\title{
Differential Responses to Beta-Adrenergic Stimulation in the Long-QT Syndrome Type 1: Characterization and Mechanisms
}

\author{
David Adolfo Sampedro-Puente ${ }^{1}$, Jesus Fernandez-Bes ${ }^{1}$, Esther Pueyo ${ }^{1,2}$ \\ ${ }^{1}$ BSICoS group, I3A, IIS Aragón, University of Zaragoza, Zaragoza, Spain \\ ${ }^{2}$ CIBER-BBN, Spain
}

\begin{abstract}
Long QT syndrome type 1 (LQT1) is caused by mutations that impair the function of the slow delayed rectifier potassium $\left(I_{K s}\right)$ channels. Most LQT1 patients experience arrhythmic events during beta-adrenergic stimulation ( $\beta$ $A S)$. A full description of the ionic mechanisms underlying arrhythmogenecity in LQT1 patients and their relation to $\beta$-AS is still lacking. In this study we constructed a set of stochastic human ventricular cell models reproducing experimental AP properties at baseline and following ionic inhibitions. Using the constructed models, we showed that AP duration, morphology and beat-to-beat variability in LQT1 are highly specific of the underlying ionic characteristics. Likewise, the response of individual $I_{K s}$-deficient cells to $\beta$-AS can range from negligible to as much as $200 \%$ increase in AP temporal variability, recognized as a marker of arrhythmogenesis in the setting of LQT1. By partial correlation analysis, major ionic factors driving $A P$ changes associated with LQT1 and $\beta-A S$ were ascertained.
\end{abstract}

\section{Introduction}

Patients affected by the long QT syndrome, a congenital disease causing abnormal QT prolongation, are at high risk of experiencing life-threatening arrhythmias. In patients with the most common variant, long QT syndrome type 1 (LQT1), 90\% of lethal events occur during physical or emotional stress, conditions associated with strong sympathetic stimulation [1]. Finding markers that anticipate pro-arrhythmic risk in the setting of LQT1 and identifying the involved mechanisms is essential to guide therapy.

Previous studies in the literature have explored proarrhythmic abnormalities in ventricular repolarization duration and morphology in LQT1 patients as well as in animal experiments of this ion channelopathy $[2,3]$. Beat-tobeat variability in the ventricular action potential (AP) and in the T-wave of the electrocardiogram in response to sympathetic stimulation has emerged as a marker of arrhyth- mic instability in LQT1 $[4,5]$. It is, however, important to note that largely varied reactions to sympathetic stimulation and arrhythmia generation have been reported in patients with the same LQT1 mutation and in animals where LQT1 has been mimicked using identical pharmacological approaches.

In this study we use stochastic electrophysiological models to investigate individual responses of human ventricular cells to $\beta$-adrenergic challenges in LQT1 and seek to determine the mechanisms underlying differential outcomes.

\section{Methods}

\subsection{AP modeling and simulation}

The O'Hara et al. (ORd) human ventricular model [6] served as a basis to construct a set of AP models covering a range of experimentally observed characteristics. The Latin Hypercube Sampling method was used to sample the conductances, denoted by $G$, of main ionic currents like $I_{K s}, I_{K r}, I_{t o}, I_{C a L}, I_{N a}$ and $I_{K 1}$ in the range of $\pm 100 \%$ their nominal value. Out of the initially generated 100 models, only those reproducing physiological steady-state AP properties at fixed pacing frequencies and the response to ionic current blockades were retained for further investigations [7-9], which made a total of 35 models. Each of these models represents a virtual cell with distinct ionic characteristics.

To investigate beat-to-beat variability of repolarization, as observed in in vivo and in vitro experiments, stochasticity was introduced into each of the constructed AP models. Specifically, the equations describing ion channel gating of $I_{K s}, I_{K r}, I_{t o}$ and $I_{C a L}$ were modified by adding a stochastic term with magnitude inversely proportional to the square root of the ion channel numbers of each ion type [10]. These channel numbers were defined by dividing the whole-cell ionic conductance in the corresponding model by the experimentally reported unitary conductance of the ion type $[11,12]$.

LQT1 was simulated by selectively blocking the $I_{K s}$ 
current at $90 \%$. $\beta$-adrenergic stimulation ( $\beta$-AS) effects on human ventricular electrophysiology were modeled using the approach described in [13], with phosphorylation levels of cellular protein kinase A (PKA) substrates calculated as in [14] and subsequent updates described in [15]. A 1 $\mu \mathrm{M}$ dose of the $\beta$-adrenergic agonist isoproterenol (ISO), producing maximal $\beta$-AS effects, was simulated.

Numerical integration was performed using the EulerMaruyama method, with a time step $d t=0.02 \mathrm{~ms}$. A total of 2500 cycles were simulated while pacing at a frequency of $1 \mathrm{~Hz}$, of which the first 500 cycles corresponded to baseline conditions, the following 500 cycles to LQT1 conditions and the final 1500 cycles to $\beta$-AS under LQT1.

\subsection{AP markers}

The following AP measures were evaluated over the last 100 cycles of each simulated condition (baseline, LQT1, LQT1 plus $\beta$-AS):

- Average AP duration at $90 \%$ repolarization (APD):

$$
\mathrm{APD}=\frac{1}{100} \sum_{n=1}^{100} \operatorname{APD}_{90}(\mathrm{n})
$$

where $\operatorname{APD}_{90}(\mathrm{n})$ is the $\mathrm{AP}$ duration at $90 \%$ repolarization for beat $n$ out of the last 100 cycles of each condition.

- Average AP triangulation (TR) calculated as

$$
\mathrm{TR}=\frac{1}{100} \sum_{n=1}^{100} \mathrm{TR}(\mathrm{n})
$$

where $\mathrm{TR}(\mathrm{n})=\mathrm{APD}_{90}(\mathrm{n})-\mathrm{APD}_{50}(\mathrm{n})$, being $\mathrm{APD}_{50}(\mathrm{n})$ the AP duration at $50 \%$ repolarization for beat $n$ out of the last 100 cycles of each condition.

- Average Short-Term Variability (STV) computed as

$$
\mathrm{STV}=\frac{1}{K} \sum_{i=1}^{K} \sum_{n=1}^{N-1} \frac{\left|\mathrm{APD}_{90}(\mathrm{n}+\mathrm{i})-\mathrm{APD}_{90}(\mathrm{n}-1+\mathrm{i})\right|}{(\mathrm{N}-1) \sqrt{2}}
$$

where $K=100-N+1$. Instantaneous STV values were calculated in N-beat windows, $\mathrm{N}=30$, and subsequently averaged over the $K$ windows comprised within the last 100 simulated cycles of each condition.

- Long-term variability (LTV) computed as

$\mathrm{LTV}=\frac{1}{K} \sum_{i=1}^{K} \sum_{n=1}^{N-1} \frac{\left|\mathrm{APD}_{90}(\mathrm{n}+\mathrm{i})+\mathrm{APD}_{90}(\mathrm{n}-1+\mathrm{i})-2 \overline{\mathrm{APD}}^{(i)}\right|}{(\mathrm{N}-1) \sqrt{2}}$ where $\overline{\mathrm{APD}}^{(i)}$ denotes the mean $\mathrm{APD}_{90}$ for the 30 beats in the window indexed by $i$.

Linear partial correlation analysis was performed to assess correlations between ionic current conductances and values of the above described AP markers while controlling for the effects of other ionic conductances [11]. Partial correlation coefficients are denoted by $\rho_{x}$, where x stands for $\triangle \mathrm{APD}, \Delta \mathrm{TR}, \Delta \mathrm{STV}$ or $\Delta \mathrm{LTV}$, representing changes in APD, TR, STV or LTV induced by $\beta$-AS under LQT1.

\section{Results and Discussions}

\subsection{AP duration and morphology}

Fig.1 presents the response of three different virtual cells to $I_{K s}$ inhibition and $\beta$-AS. $\operatorname{APD}_{90}(\mathrm{n})$ (top panel) and $\mathrm{TR}(\mathrm{n})$ (bottom panel) are shown during baseline, LQT1 and LQT1 $+\beta$-AS. As can be observed from the graphics, APD and TR are larger in LQT1 than at baseline for the three illustrated cells. However, the degree of APD and TR augmentation is notably dependent on the evaluated cell. For the cell shown in black, a very large APD prolongation accompanied by AP triangulation can be noted, while the change induced by $I_{K s}$ inhibition in the other two cells is minor.
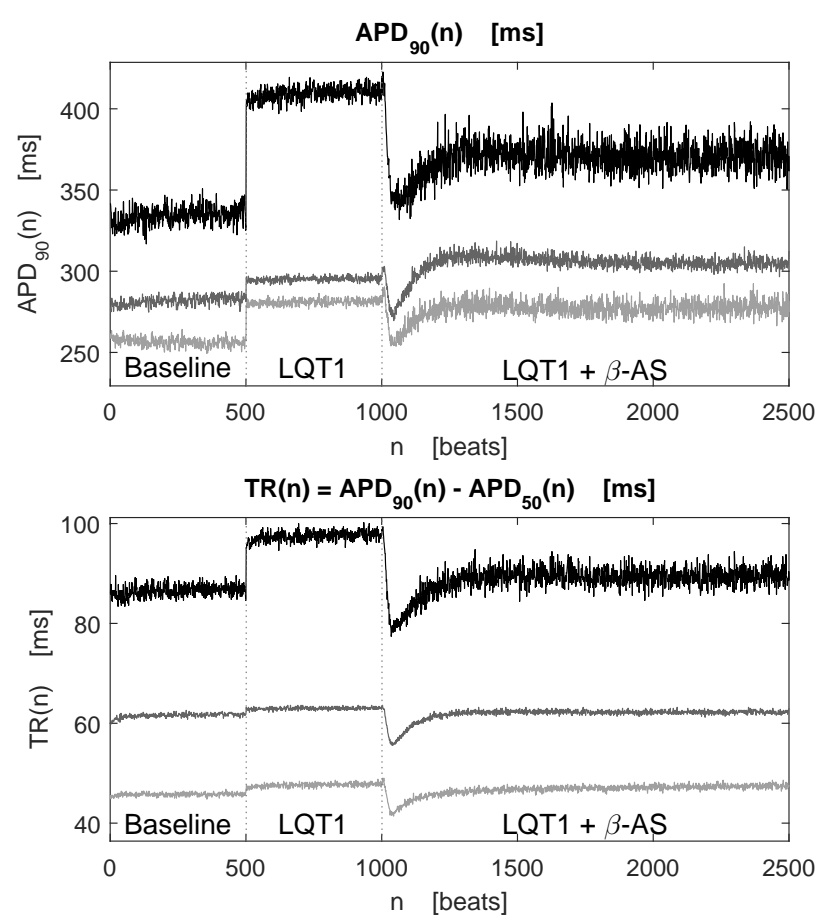

Figure 1. $\mathrm{APD}_{90}(\mathrm{n})$ and $\mathrm{TR}(\mathrm{n})$ values for three simulated cells with distinct ionic characteristics. Vertical dotted lines are shown to delimit the time periods corresponding to baseline, LQT1 and LQT1 $+\beta$-AS conditions. 

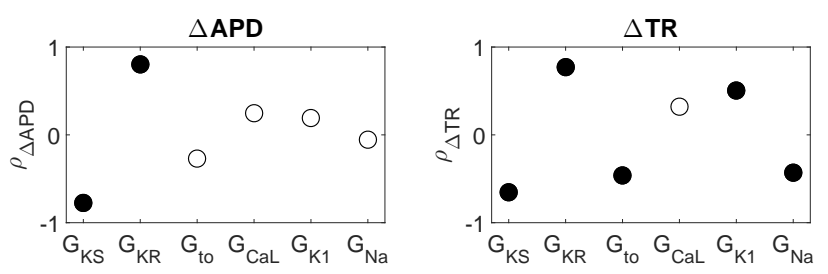

Figure 2. Left panel: Partial correlation coefficients between $\triangle \mathrm{APD}$ and ionic current conductances, with black circles indicating significant correlation. Right panel: similarly for $\Delta \mathrm{TR}$.

ISO application caused a sudden transient drop in both $\mathrm{APD}_{90}(\mathrm{n})$ and $\mathrm{TR}(\mathrm{n})$ for the three illustrated cells, which was followed by slower increases in the two AP markers. Again, the degree of change in APD and TR due to $\beta$ AS under LQT1 conditions was significantly different for the three cells, ranging from negligible to very large increases/decreases, particularly regarding APD.

Fig. 2 shows the results of the partial correlation analysis when evaluating the differences in APD (TR, respectively) between LQT1 $+\beta$-AS and LQT1. $\triangle \mathrm{APD}$ was found to be significantly positively correlated with $G_{K r}$, but significantly negatively correlated with $G_{K s}$. This means that LQT1 cells with strong $I_{K r}$ upregulation or $I_{K s}$ downregulation are expected to experience very large APD changes in response to $\beta$-AS. In the case of $\Delta \mathrm{TR}$, significantly negative or positive correlations were found with all current conductances except for $G_{C a L}$. This indicates that upregulation of $I_{K r}$ or $I_{K 1}$ and downregulation of $I_{K s}, I_{t o}$ or $I_{N a}$ are all expected to have a large independent impact on AP triangulation.

\subsection{Beat-to-beat variability}

Results of temporal $\mathrm{APD}_{90}$ variability are presented in Fig. 3 for the same three virtual cells illustrated in 3.1. In the left column, Poincaré plots are presented for baseline, LQT1 and LQT1 + $\beta$-AS conditions, with APD values calculated over the last 100 cycles of each simulated condition. Corresponding STV measures are shown in the right column for the three virtual cells. As can be observed from Fig.3, STV experienced mild to moderate changes in response to $I_{K s}$ inhibition $\left(\triangle \mathrm{STV}_{L Q T 1}\right)$, with percentages of change across all simulated cells varying from $-45.4 \%$ to $20 \%$.

The change in STV due to $\beta$-AS effects was even more varied across the simulated population of cells. While many cells, like those shown in Fig.3, presented an important increase in STV (reaching a maximum of 214\%), there were cells where STV barely changed or even slightly decreased. It is of relevance to note that changes in STV due to either $I_{K s}$ inhibition or $\beta$-AS were independent of APD.
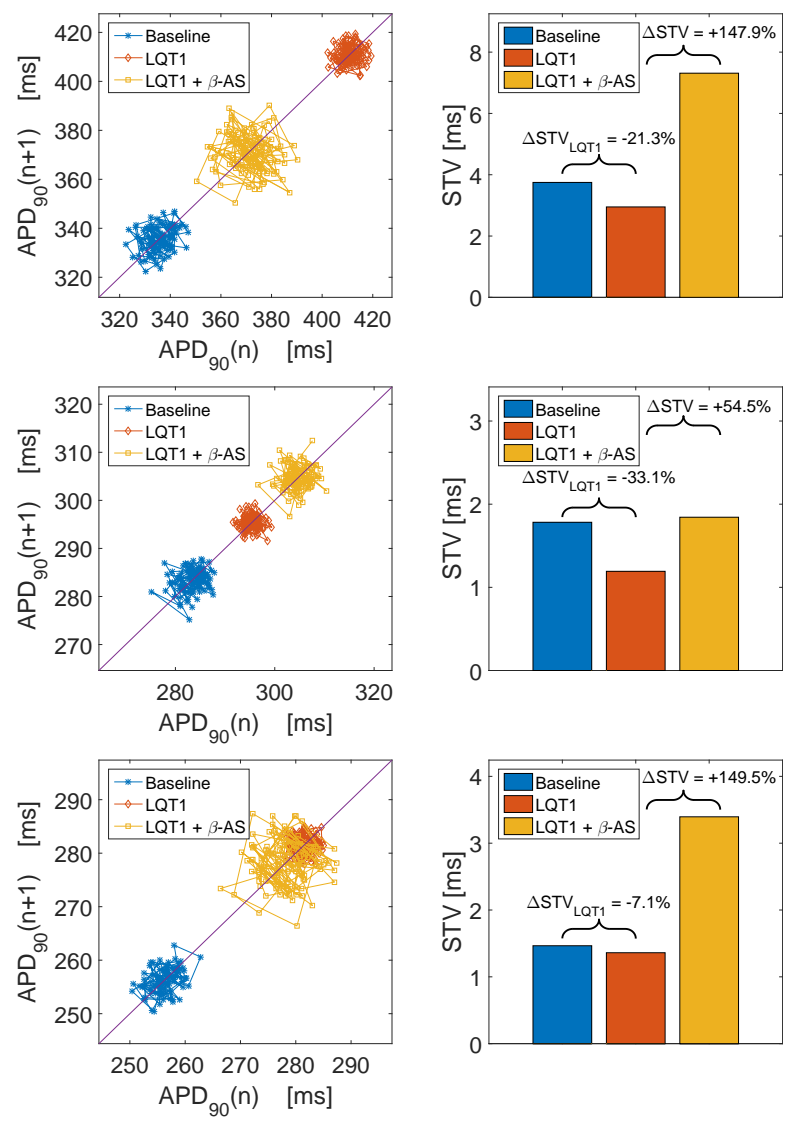

Figure 3. Left: Poincaré plots for three virtual cells under baseline (blue), LQT1 (red) and LQT1 + $\beta$-AS (yellow). Right: Corresponding STV values and percentages of change due to $I_{K s}$ inhibition and $\beta$-AS.

As an example, the second and third cells represented in Fig.3 had similar APD values at baseline, whereas STV decreases associated with $I_{K s}$ inhibition were of $33 \%$ and $7 \%$, respectively. Likewise, those two cells shared similar APD values for LQT1 conditions, but the increments in STV associated with $\beta$-AS effects were of $55 \%$ and $150 \%$, respectively. This highly inter-individual response of STV to LQT1 and a subsequent adrenergic action was similarly observed for LTV.
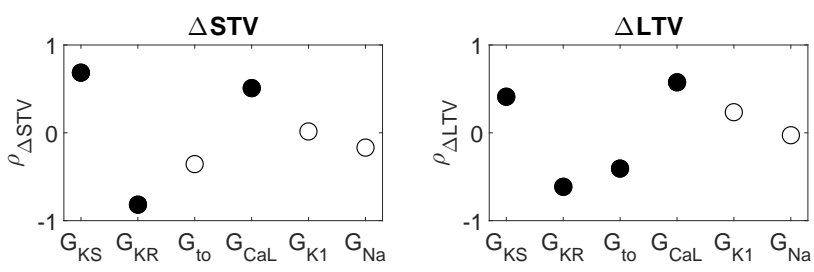

Figure 4. Left panel: Partial correlation coefficients between $\triangle \mathrm{STV}$ and ionic current conductances, with black circles indicating significant correlation. Right panel: similarly for $\triangle \mathrm{LTV}$. 
Partial correlation results for the differences in STV and LTV between LQT1 + $\beta$-AS and LQT1 are shown in Fig.4. STV was found to be significantly positively correlated with $G_{K s}$ and $G_{C a L}$ but significantly negatively correlated with $G_{K r}$. Similarly occurred for LTV, with an additional significantly negative correlation with $G_{t o}$. The key role of $I_{K s}$ and $I_{C a L}$ in modulating STV and LTV response to $\beta$-AS can be attributed to the large increases in these two currents induced by PKA phosphorylation.

\section{Conclusions}

By building a set of stochastic human ventricular cell models representing experimental AP properties, the response of individual cells to LQT1 and $\beta$-AS were characterized. Very heterogeneous changes in APD, triangulation and temporal variability were observed depending on the ionic cell characteristics. For some cells, $\beta$-AS under LQT1 had practically no effect on the AP, while for other cells increases larger than $200 \%$ were quantified, particularly regarding beta-to-beat variability. Key ionic determinants underlying the nature of the AP response to an adrenergic action in the presence of LQT1 were determined.

\section{Acknowledgements}

This work was supported by projects TIN2013-41998$\mathrm{R}$ and DPI2016-75458-R from MINECO, Spain, MULTITOOLS2HEART from CIBER-BBN through ISCIII, Spain, European Social Fund (EU) and Aragón Government through BSICoS group (T96) and a personal grant to D. A. Sampedro-Puente, and by the European Research Council (ERC) through project ERC-2014-StG 638284. Computations were performed by ICTS NANBIOSIS (HPC Unit at University of Zaragoza).

\section{References}

[1] Schwartz PJ, Priori SG, Spazzolini C, Moss AJ, Vincent GM, Napolitano C, Denjoy I, Guicheney P, Breithardt G, Keating MT, Towbin JA, Beggs AH, Brink P, Wilde AA, Toivonen L, Zareba W, Robinson JL, Timothy KW, Corfield V, Wattanasirichaigoon D, Corbett C, Haverkamp W, Schulze-Bahr E, Lehmann MH, Schwartz K, Coumel P, Bloise R. Genotype-phenotype correlation in the long-QT syndrome: Gene-specific triggers for life-threatening arrhythmias. Circulation 2001;103:89-95.

[2] Liu GX, Choi BR, Ziv O, Li W, de Lange E, Qu Z, Koren G. Differential conditions for early after-depolarizations and triggered activity in cardiomyocytes derived from transgenic LQT1 and LQT2 rabbits. J Physiol 2012;590:11711180.

[3] Jons C, O-Uchi J, Moss AJ, Reumann M, Rice JJ, Goldenberg I, Zareba W, Wilde AAM, Shimizu W, Kanters JK, McNitt S, Hofman N, Robinson JL, Lopes CMB. Use of mutant-specific ion channel characteristics for risk stratification of long QT syndrome patients. Sci Transl Med 2011; 3:76ra28.

[4] Satomi K, Shimizu W, Takaki H, Suyama K, Kurita T, Aihara N, Kamakura S. Response of beat-by-beat QT variability to sympathetic stimulation in the LQT1 form of congenital long QT syndrome. Heart Rhythm 2005;2:149-154.

[5] Gallacher D, Van de Water A, Van der Linder H, Hermans A, Lu H, Towart R, Volders P. In vivo mechanisms precipitating torsades de pointes in a canine model of drug-induced long-QT1 syndrome. Cardiovasc Res 2007;76:247-256.

[6] O’Hara T, Virág L, Varró A, Rudy Y. Simulation of the undiseased human cardiac ventricular action potential: Model formulation and experimental validation. PLoS Comput Biol 2011;7:e1002061.

[7] Jost N, Varro A, Szuts V, Kovacs PP, Seprényi G, Biliczki P, Lengyel C, Prorok J, Bitay M, Ördög B, Szabad J, VargaOrvos Z, Puskas L, Cotella D, Papp JG, Virag L, Nattel $\mathrm{S}$. Molecular basis of repolarization reserve differences between dogs and man. Circulation 2008;118:342.

[8] O'Hara T, Rudy Y. Quantitative comparison of cardiac ventricular myocyte electrophysiology and response to drugs in human and nonhuman species. Am J Physiol Heart Circ Physiol 2012;302:H1023-H1030.

[9] Grandi E, Pasqualini FS, Bers DM. A novel computational model of the human ventricular action potential and $\mathrm{Ca}$ transient. J Mol Cell Cardiol 2010;48:112-121.

[10] van. Kampen NG. Stochastic processes in physics and chemistry. Elsevier, 2007.

[11] Pueyo E, Dangerfield CE, Britton OJ, Virág L, Kistamás K, Szentandrássy N, Jost N, Varró A, Nánási PP, Burrage K, Rodríguez B. Experimentally-based computational investigation into beat-to-beat variability in ventricular repolarization and its response to ionic current Inhibition. PLoS One 2016;11:e0151461.

[12] Pueyo E, Corrias A, Virág L, Jost N, Szél T, Varró A, Szentandrássy N, Nánási PP, Burrage K, Rodríguez B. A multiscale investigation of repolarization variability and its role in cardiac arrhythmogenesis. Biophys J 2011;101:28922902.

[13] Pueyo E, Orini M, Rodríguez JF, Taggart P. Interactive effect of beta-adrenergic stimulation and mechanical stretch on low-frequency oscillations of ventricular action potential duration in humans. J Mol Cell Cardiol 2016;97:93-105.

[14] Soltis AR, Saucerman JJ. Synergy between CaMKII substrates and $\beta$-adrenergic signaling in regulation of cardiac myocyte Ca2+ handling. Biophys J 2010;99:2038-2047.

[15] Xie Y, Grandi E, Puglisi JL, Sato D, Bers DM. $\beta$-adrenergic stimulation activates early afterdepolarizations transiently via kinetic mismatch of PKA targets. J Mol Cell Cardiol 2013;58:153-161.

Address for correspondence:

David Adolfo Sampedro-Puente

Instituto de Investigación en Ingeniería de Aragón (I3A). Lab. 4.0.5. C/ Mariano Esquillor s/n, 50018, Zaragoza.

sampedro@unizar.es 